Supporting Materials for "Masterless Soft Lithography: The Investigation and Patterning of UVO Adhesion on PDMS Surfaces." By William R. Childs, Michael J. Motala, Keon Jae Lee, and Ralph G. Nuzzo.

\title{
Supplemental Results
}

UVO Spacing Effects for Photomask Design Rules

Early attempts to adhesively transfer PDMS using contact photolithography were unsuccessful, but clearly demonstrated the necessity of maintaining a sample-mask separation for successful PDMS transfer. In a previous publication, PDMS stamps for DTL were typically flood exposed $\sim 1 \mathrm{~mm}$ from an ozone-active, low-pressure mercury UV source to induce surface modifications that lead to strong adhesive bonds. When stamps were withdraw from the bulb by distances larger than $\sim 1 \mathrm{~cm}$, the surface chemistry changed and no adhesion was observed. However, PDMS stamps held at a distance of $3 \mathrm{~cm}$ from the bulb and irradiated through a quartz slide by the same mercury lamp, while maintaining a spacing of a millimeter between the quartz slide and the PDMS sample, were capable of being bound irreversibly to an oxide-bearing substrate. No adhesion was observed, however, when the PDMS stamp was held in direct contact with the quartz slide during the irradiation process (times ranging from 1-30 minutes). As expected, adhesion could also be prevented, while maintaining proper separation of the quartz slide and sample, by coating the quartz slide with a $100 \mathrm{~nm}$ thick gold film to block UV transmission. These results confirm that a small air gap is necessary to effect a surface modification that produces strong adhesion of PDMS to another substrate.

The necessity of a patterned shim was illustrated by another experiment where a glass slide was patterned with an array of oblate holes, with dimensions of $\sim 100 \times 100$ 
$\mu \mathrm{m}$, in a $100 \mathrm{~nm}$ thick gold coating. To provide an appropriate air gap of $\sim 40 \mu \mathrm{m}$, an open shim framework was constructed by cutting away the center of a Mylar sheet, and placing the resulting, ring-shaped Mylar shim between the PDMS stamp and the patterned gold slide during a UV exposure from a conventional Hg-discharge lamp. The exposed sample, patterned with a square array of $2 \mu \mathrm{m}$ diameter PDMS posts, was placed into contact with a glass substrate, and heated according to the standard protocol used to effect adhesion. An irregular pattern transfer was found to result as shown in Figure S1a. The modulated character of this pattern transfer was highly periodic, varying on a scale consistent with the $100 \mu \mathrm{m}$ openings of the exposure mask, which suggested to us that multiple reflections near the mask feature edges were strongly impacting the pattern transfer. This assumption prompted us to adopt the UVO photomask design presented in the main text, which in conjunction with a new UV exposure source, led to dramatically improved results.

We examined the specific effect of the photoresist shim thickness on the character of UVO induced pattern transfer using another experiment. Three UVO photomasks were fabricated with the same square array of $45 \mathrm{x} 55 \mu \mathrm{m}^{2}$ transmissive regions, but differing in the thickness of the photoresist shim employed: masks with thickness values of $100 \mathrm{~nm}, 3 \mu \mathrm{m}$, and $17 \mu \mathrm{m}$ were examined for this study. Three identically patterned PDMS stamps having a square array of posts were exposed to the deuterium source for four minutes, each in conjugation with one of the photomasks, and then used to pattern test glass substrates by following a series of identical steps. The PDMS pattern transferred from a stamp exposed with the $100 \mathrm{~nm}$ thick mask was composed of a few PDMS posts in regularly spaced domains, but the sizes of the domains were irregular and 
generally much smaller than the area of exposure [Figure S1b]. The stamp exposed through the $3 \mu \mathrm{m}$ thick photomask performed better, transferring the oblate array of PDMS posts in domains consistent with the size and spacing of the areas of irradiation [Figure S1c]. Defects were common in these patterns and numerous irregular patches were seen where the PDMS posts failed to transfer. The PDMS stamp that was irradiated through the UVO photomask with a $17 \mu \mathrm{m}$ thick spacer transferred a square array of PDMS posts in domains which contained no observable flaws, as illustrated by the data shown in Figure S1d. Stamps irradiated by a mercury source through each of these masks for identical times produced similar results, except that the PDMS posts transferred were typically much thinner $(<20 \mathrm{~nm}$, data not shown).

\section{UVO Irradiation Time Dependence of P-CMF}

We examined the effect of the irradiation time on pattern transfer by exposing similar PDMS stamps to a deuterium lamp for various durations. In this experiment, the mask spacing was maintained at $17 \mu \mathrm{m}$, and the period of irradiation by the deuterium bulb was varied from 3 to 20 minutes. A PDMS stamp exposed for three minutes transferred a square array of PDMS posts in well defined domains composed of thick posts, but an irregular number of thinner posts were also observed for most of the irradiated regions as illustrated by the data shown in Figure S2a (where an inset micrograph of the UVO photomask at equal magnification is also provided). Identically patterned stamps were irradiated similarly through this photomask for periods of 8 or 20 minutes. The PDMS stamp irradiated for eight minutes transferred a square array of thick PDMS posts in well defined domains with few defects or thin posts being evidenced as shown in Figure S2b. The PDMS stamp irradiated for 20 minutes transferred a PDMS 
pattern showing the same oblate arrays of circular PDMS posts; some degradation was observed, however, towards the center of the pattern where the irradiation was most intense [Figure S2e].

\section{Surface Topography of Stamps used for DTL patterning}

An AFM scan was taken of a PDMS stamp that had been used to transfer a PCMF pattern similar to that shown in Figure S2a to elucidate the nature of this mode of pattern transfer. Figure S3a shows an AFM image of a region of a stamp that illustrates an important feature of DTL transfer corresponding to a transfer similar to those in Figure 3 of the main text. The thin PDMS features arise as a result of a cohesive failure in the bound glass-stamp structure that, upon pealing, transfers the tip of the post to the glass surface. The taller posts, however, are torn physically from the bulk of the stamp. The effected region is illustrated by the representative height contours seen in the line scan of Figure S3b. It is this transfer of the entire post with the additional, consistent transfer of material from the bulk of the stamp that allow for P-CMF to transfer microstructures that exceeded the height of the features originally patterned on the PDMS stamp.

A similar AFM scan was taken of a stamp that had been used to transfer the UVO Patterned Adhesive Transfer pattern shown in Figure 4a of the text. The correlated AFM images of the PDMS stamp used for this pattern transfer showed an identical, but inverted pattern consisting of $\sim 800 \mathrm{~nm}$ deep trenches with openings $4 \mu \mathrm{m}$ wide and a spacing of $4 \mu \mathrm{m}$ as illustrated by the AFM images shown in Figure S3c. The line scan shown in Figure S3d provides a representative illustration of the high fidelity of the pattern transfer achieved throughout the area of exposure. 
One Step Multiple Exposure UVO Induced Adhesive Transfer

Sylgard 184 was cured for a minimum of 24 hours at $70^{\circ} \mathrm{C}$. The unpatterned PDMS was placed in contact with a UVO exposure mask patterned with $3 \mu \mathrm{m}$ lines separated by identical spacings. This assembly was then irradiated for 30 minutes at a distance of $\sim 1 \mathrm{~cm}$ from a deuterium source. After exposure, the PDMS stamp was peeled away from the photomask, rotated by $90^{\circ}$, and placed back into contact with the photomask. A second 30 minute deuterium exposure was then preformed. Upon completion of the second exposure, the PDMS slab was removed from the UVO mask and placed face down into contact with a glass slide. The assembly was heated in an oven at $70^{\circ} \mathrm{C}$ overnight. Starting from a corner the PDMS was then removed yielding a PDMS patterned grid on the glass as shown in Figure S4.

ToF-SIMS Surface Characterization of Modified and Unmodified PDMS

To develop a mechanistic understanding of the oxidative chemistries that mediate pattern transfer, we examined secondary ion mass spectra from a PDMS surface modified with a standard UVO adhesion protocol, and compared these results to ones obtained from a control. Samples from a single batch of PDMS were cast by spinning onto silicon wafers and cured at $70^{\circ} \mathrm{C}$ for $16 \mathrm{hrs}$. A quartz slide was placed over the PDMS film with a $40 \mu \mathrm{m}$ spacer prior to irradiation by either the deuterium or mercury sources as described in the main experimental section.

Negative ToF-SIMS spectra were recorded for each sample (and a control) using identical acquisition times and conditions. At low resolution, the spectra plotted over a mass range of 30-100 m/z, exhibited few apparent differences as shown in Figure S5. ToF-SIMS Characterization of the UVO Oxidative Adhesion Mechanism 
We examined individual component reactants that appeared to be required for a UVO modification to produce a strong adhesive bond and exposed PDMS films to these species under varying conditions while simultaneously characterizing their individual and combined effects by ToF-SIMS. PDMS films from the same batch of polymer were cast onto five silicon wafers and cured. Five unpatterned bulk PDMS slabs were also prepared from the resin. These formed five sets of "bonding" pairs used to collect the data shown in Figure S6. Figure S6a corresponds to a PDMS coated wafer and PDMS "stamp" that were both exposed simultaneously to UV from a mercury source through a quartz slide held $1 \mathrm{~mm}$ from the PDMS surface for 4 minutes, a condition that produces strong adhesion. The PDMS coated wafer was analyzed by ToF-SIMS, and the PDMS slab was placed into contact with a clean glass slide and heated to $70^{\circ} \mathrm{C}$ for at least 20 minutes. As expected, strong adhesion of the PDMS resulted. This process was repeated for the remaining samples under four separate sets of conditions: the data in Figure S6b corresponds to a slab irradiated by filtered UV for 4 minutes (both film and stamp were irradiated by UV through a quartz slide that had been coated with a thick, cured PDMS film as a filter to block wavelengths $<230 \mathrm{~nm}$ ); the data in Figure S6c correspond to a film exposed for 4 minutes to ozone generated using an electrical source; while that in Figure S6d was based on an exposure that combined the ozone ambient with the filtered UV radiation (>230 $\mathrm{nm}$ ) for 4 minutes. Figure S6e is a spectrum taken using an untreated control sample.

Adhesion to glass was found for the correlated slab samples in only two cases, those corresponding to the treatments of Figures S6a and d. These data establish that the UVO adhesion is strongly correlated with the appearance of the $\mathrm{m} / \mathrm{e}=45.00$ peak in the 
ToF-SIMS data. We also established the pattern of implicated photochemistry when one notes the optical properties of PDMS, a material that strongly absorbs UV light at wavelengths below $230 \mathrm{~nm}$. Ozone is efficiently generated by the photoexcitation of oxygen at wavelengths near $\sim 185 \mathrm{~nm}$. The uncoated quartz slides used in this work are transmissive at this wavelength. The exposure of Figure S6a generates ozone while that of $6 \mathrm{~b}$ does not. An ozone exposure, of itself, however, is insufficient to produce strong adhesion (as evidenced by the treatment corresponding to Figure S6c). Ozone is susceptible to photochemical decomposition via processes that become facile at excitation wavelengths below $\sim 254 \mathrm{~nm}$. The PDMS coated quartz optics are transmissive at this range, yet adhesion is only observed in this instance when ozone is present from an outside source (the conditions of exposure corresponding to Figure S6d). Taken together with the observation that UV irradiation at essentially any wavelength between 180 and $250 \mathrm{~nm}$ in the absence of oxygen is ineffective at producing a bondable PDMS surface, a strong set of inferences is raised regarding the underlying mechanisms involved. The data presently available strongly suggest that reactive species derived from the photodecomposition of ozone (eg. $\mathrm{O}, \mathrm{O}_{2}$, etc...) are likely involved. The combination of atmospheric reactants with the molecular constituents of PDMS in a near surface region must then, in turn, lead to chemical reactions that foster the strong bonding. These species appear to be correlated with the $\mathrm{m} / \mathrm{e}=45.00$ fragment seen in the SIMS data. 


\section{Figures}

Figure 1: Identically molded PDMS stamps were irradiated under identical conditions through UVO photomasks which differed only in the shim thickness of the photomask spacer. The resulting PDMS patterns that were transferred onto glass, using UVO masks

with the shim thicknesses indicated, are shown in the corresponding optical micrographs: (a) $40 \mu \mathrm{m}$ (open Mylar shim with conventional UV photomask) (b) $100 \mathrm{~nm}$, (c) $3 \mu \mathrm{m}$, and (d) $17 \mu \mathrm{m}$. (An identical optical aberration is in the background of each image that is not indicative of the samples).

Figure 2: PDMS stamps were irradiated through the same UVO photomask for various times and transferred onto glass surfaces as seen in these optical micrographs: (a) 3 minutes; (b) 8 minutes; and (c) 20 minutes. A picture of the UVO photomask used for patterning is inset to scale in picture (a).

Figure 3: AFM scans of PDMS transfer tools that had already been used to transfer a PDMS pattern onto a surface using either a P-CMF procedure ( $a$ and $b$ )or a UVO Patterned Adhesive Transfer procedure (c and d).

Figure 4: AFM scans of a grid pattern that was transferred onto glass after a single PDMS slab was exposed in two steps through a UVO photomask patterned only with a single set of parallel lines; the mask was merely rotated $90^{\circ} \mathrm{C}$ between exposures. The lines scans were measured along orthogonal directions.

Figure 5: Negative ion ToF-SIMS spectra of PDMS surfaces that were (a) unmodified, or modified by either irradiation from (b) mercury or (c) deuterium source under UVO conditions for 4 minutes. 
Figure 6: Negative ion ToF-SIMS spectra of PDMS surfaces under the following conditions: (a) irradiated by a mercury lamp through a quartz slide for 4 minutes (UVO condition); (b) irradiated by a mercury lamp through a PDMS coated ( $\geq 230 \mathrm{~nm}$ ) quartz slide for 4 minutes (non-UVO condition); (c) exposed to ozone in oxygen for 4 minutes (non-UVO condition); (d) irradiated by a mercury lamp through a PDMS coated $(\geq 230$ $\mathrm{nm}$ ) quartz slide for 4 minutes while exposed to ozone in oxygen (UVO condition); and (e) not irradiated by UV or exposed to ozone (control). 